\title{
Pattern Recognition and Information Fusion Using Belief Functions: Some Recent Developments
}

\author{
Thierry Denœux \\ HEUDIASYC, UTC, CNRS \\ Centre de Recherche de Royallieu \\ BP 20529, F-60205 Compiègne, France
}

The Transferable Belief Model (TBM) is a general framework for reasoning with uncertainty using belief functions [8]. Of particular interest is the General Bayesian Theorem (GBT), an extension of Bayes's theorem in which probability measures are replaced by belief functions, and no prior knowledge is assumed [76].

Until recently, applications of the GBT have been fairly limited, mainly because of lack of methods for constructing belief functions from observation data. The availability of such methods [421] as well as new combination rules for merging partially overlapping items of evidence [5] now extend the applicability of the TBM to a wider class of statistical pattern recognition and information fusion tasks. These recent developments will be reviewed, and applications to various problems such as novelty detection [3] and partially supervised learning using mixture models will be discussed.

This talk is mostly self-contained. Relevant material (papers, slides) can be found at http: //www . hds . utc . fr ${ }^{\sim}$ tdenoeux.

\section{References}

1. Aregui, A., Denœux, T.: Consonant belief function induced by a confidence set of pignistic probabilities. In: Proceedings of ECSQARU '2007, Hammamet, Tunisia (to appear)

2. Aregui, A., Denœux, T.: Constructing predictive belief functions from continuous sample data using confidence bands. In: De Cooman, G., Vejnarová, J., Zaffalon, M. (eds.) Proceedings of the Fifth International Symposium on Imprecise Probability: Theories and Applications (ISIPTA '07), July 2007, Prague, Czech Republic, pp. 11-20 (2007)

3. Aregui, A., Denœux, T.: Fusion of one-class classifiers in the belief function framework. In: Proceedings of the 10th Int. Conf. on Information Fusion, Canada (July 2007)

4. Denœux, T.: Constructing belief functions from sample data using multinomial confidence regions. International Journal of Approximate Reasoning 42(3), 228-252 (2006)

5. Denœux, T.: Conjunctive and disjunctive combination of belief functions induced by non distinct bodies of evidence. Artificial Intelligence 2007 (in press)

6. Denœux, T., Smets, P.: Classification using belief functions: the relationship between the casebased and model-based approaches. IEEE Transactions on Systems, Man and Cybernetics B 36(6), 1395-1406 (2006)

7. Smets, P.: Belief functions: the disjunctive rule of combination and the generalized Bayesian theorem. International Journal of Approximate Reasoning 9, 1-35 (1993)

8. Smets, P., Kennes, R.: The Transferable Belief Model. Artificial Intelligence 66, 191-243 (1994) 